\title{
Therapeutic use of rTMS
}

\author{
Saxby Pridmore
}

A recent Perspective article ${ }^{1}$ on the therapeutic uses of repetitive transcranial magnetic stimulation (rTMS) argues that, before clinical applications such as the treatment of major disorders are attempted, "much more transparent hypotheses" are required, and that studies of "the pathological processes underlying the conditions for which it is used will reveal whether rTMS really does offer therapeutic potential".

The problem is that we have patients now who are suffering and committing suicide, and we are decades away from fully understanding the pathological processes of major depression. As the authors point out, guidelines to reduce side effects have been "remarkably successful", and the treatment must now be considered relatively safe. The alternative treatment is stigmatizing electroconvulsive therapy, which involves general anaesthetic, convulsion and temporary memory disorder.

It should be acknowledged that the pathophysiology of psychiatric disorders is poorly understood, and that most important therapeutic advances have been serendipitous rather than hypothesis-driven findings. Chlorpromazine, the first antipsychotic, was observed to be useful when it was used as an anaesthetic agent in people with schizophrenia. Iproniazid, the first antidepressant, was found to improve mood when used as an anti-tuberculous agent. Electroconvulsive therapy was hypothesis-driven, but the hypothesis and the disorder were wrong. Electroconvulsive therapy was first used in the treatment of schizophrenia, rather than depression, because it was believed that epilepsy and schizophrenia were mutually exclusive conditions.

The Perspective article describes two models that underpin the use of rTMS in the treatment of depression: a 'repair' and an 'interaction' model. I myself use a third: the 'a little bit of a good thing' model. Electroconvulsive therapy is the most efficacious antidepressant, but it has many drawbacks. As rTMS is relatively safe and can place small amounts of electrical current in a region that is known to be under-active in depression (and which is connected to other structures that are involved in this disorder), I have no hesitation in providing this treatment. In one third of cases the results are excellent.

1. Ridding, M. \& Rothwell, J. Is there a future for therapeutic use of transcranial magnetic stimulation? Nature Rev. Neurosci. 8, 559-567 (2007).

Department of Psychiatry, University of Tasmania, Royal Hobart Hospital, Hobart, Tasmania 7000, Australia.e-mail: spridmore@iprimus.com.au 